EGU21-15468

EGU General Assembly 2021

(c) Author(s) 2021. This work is distributed under

the Creative Commons Attribution 4.0 License.

\title{
Large-Eddy Simulation in LSPIV techniques: the study of surface turbolence
}

\author{
Francesco Alongi, Giuseppe Ciraolo, Enrico Napoli, Dario Pumo, and Leonardo V. Noto \\ Università degli studi di Palermo, Dipartimento di Ingegneria, Palermo, Italy (francesco.alongi01@unipa.it)
}

In recent years, technological advances have been observed in environmental monitoring field, leading to a rapid spread of innovative technologies overcoming many historical challenges. In river monitoring field the use of image-based techniques provides non-intrusive measurements ensuring the best safety conditions for operators. The most used optical methods are the LargeScale Particle Image Velocimetry (LSPIV) and the Large-Scale Particle Tracking Velocimetry (LSPTV).

In LSPIV and LSPTV techniques a floating tracer is introduced on the water surface and its motion is recorded by commercial devices (e.g. digital cameras). Resulting videos are then processed by free and open source software which applies a statistical cross-correlation analysis to provide the instantaneous surface velocity field.

The aim of this work is to investigate the performance of the most widely used LSPIV software in estimating the surface velocity field taking into account the presence of turbulent structures. Indeed a typical feature of natural river is the presence of turbulent eddies which makes the tracer patterns above the water surface difficult to predict. The evaluation of tracer particle displacement is further complicated by the negative phenomenon of aggregation; it influences cross-correlation causing an incorrect estimation of the velocity vectors.

The study of the hydraulic turbulence of a natural river has been tackled from a numerical point of view. PANORMUS (Parallel Numerical Open-Source Model for Unsteady Flow Simulations) package (Napoli, 2011) has been used by adopting a LES (Large Eddy Simulation) scheme. PANORMUS is a numerical tool coded to solve the 3D momentum equations for incompressible flows (NavierStokes and Reynolds equations) using the Finite-Volume Method (FVM). The analyses were carried out on real cases modelled with PANORMUS-LES package. The hydraulic reconstructed domains are characterised by regular cross sections, accurately derived from real topographic survey campaigns, and low river-bed roughness (smooth concrete surface).

Synthetic sequences of tracer motion were derived from the hydraulic model and then processed by using LSPIV software.

The results of such numerical analyses have allowed an evaluation of LSPIV performance assessing the errors in terms of mean value of the surface velocity field and velocity along transverse transects. 